\title{
Carbon Precursor Structures and Graphene on Palladium Nanoparticles
}

\author{
Clemens Barth* \\ Aix-Marseille Université, CNRS, CINaM UMR 7325, 13288 Marseille, France \\ E-mail: barth@cinam.univ-mrs.fr
}

${ }^{*}$ To whom correspondence should be addressed 


\begin{abstract}
Due to their stable catalytic properties graphene-encapsulated metal nanoparticles (NPs) have come into the focus of heterogeneous catalysis. Research on graphene's atomic structure is a prerequisite for understanding and controlling catalytic processes, however, it is still at its very beginning considering metal NPs. This atomic-scale scanning tunnelling microscopy study documents preliminary growth stages and the structural properties of graphene on HOPG (highly oriented pyrolytic graphite) supported palladium NPs. It is shown that an annealing of the NPs at $\sim 650^{\circ} \mathrm{C}$ in a few tens of Langmuir of ethylene (ethene) leads first to a carbon precursor structure covering the NP's (111) facets. At higher ethylene exposures, the precursor structure turns into single-layer graphene encapsulating the NPs. Graphene encapsulated palladium NPs with a size larger than $\sim 30 \mathrm{~nm}$ exhibit mostly one single and almost defect-free graphene sheet on a facet. Such a perfect growth does not take place on the facets of smaller NPs where several graphene nanosheets and defects are generally observed.
\end{abstract}

\title{
Introduction
}

Carbon either adsorbed on or absorbed inside metal nanoparticles (NPs) plays a major role in heterogeneous catalysis, in particular with respect to catalyst de-activation ${ }^{1}$, hydrogenation reactions ${ }^{2,3}$, the Fischer-Tropsch synthesis ${ }^{4}$ and the synthesis of carbon nanotubes ${ }^{5}$. Since a few years, carbon can be synthesized in the form of graphene directly on metal NPs by annealing a precursor material ${ }^{6,7,8,9}$, pulsed Laser deposition ${ }^{10}$ or chemical vapor deposition $(\mathrm{CVD})^{11,12}$. Such core-shell graphene encapsulated NPs (G@NPs) have very interesting properties for applications in electrocatalysis, in particular with respect to cheap and reactive NP material: thanks to the protecting graphene shell a long-term activity and high stability 
against degradation in several environments is achieved ${ }^{8,9,12,13}$. Even more, the graphene-NP ensemble optimizes the electronic structure of graphene and thereby triggers the catalytic activity on the inert graphene surface ${ }^{12}$. Since recently, G@NPs are also believed to form a new class of catalysts that lead to catalysis under cover ${ }^{14,15}$ : the graphene wall and NP facets form a nanocontainer, in which reactions take place as demonstrated recently with G@PtNPs ${ }^{7,16}$.

Important in all such G@NP related studies is the characterization of the NP's morphology (e.g., the thickness of graphene), which is mostly accomplished by transmission electron microscopy ${ }^{6,7,8,9,10,11,12}$. However, equally important is an atomic-scale characterization of the graphene structure with an access to defects and the edges, which both play a large role in electrocatalysis and in particular in catalysis under cover ${ }^{14,15}$ : for instance, in the latter field basic reactant or product molecules like oxygen, hydrogen, $\mathrm{CO}$ and water can enter or leave the nanocontainer by passing through the defects and edges of graphene ${ }^{7}$.

Metal supported graphene can be unambiguously identified by scanning tunnelling microscopy (STM), which permits analyzing the atomic structure and defects in great detail as exemplified on single-crystal metal surfaces ${ }^{17,18,19}$. However, despite the large demand to study G@NPs in catalysis ${ }^{14,15}$ there is only one STM work so far that deals with G@NiNPs ${ }^{20}$.

In the work here, graphene encapsulated palladium NPs (G@PdNPs) grown on the highly oriented pyrolytic graphite (HOPG) surface are considered. Palladium is a member of the platinum group metals (PGM) and is therefore an important material in heterogeneous catalysis. Even more, it is a very suitable material to study phenomena of subsurface carbon $^{21,22,23,24,25,26,27,28,29}$ thanks to its high carbon solubility ${ }^{30}$. Graphene was characterized only once on PdNPs by TEM and Raman spectroscopy ${ }^{31}$ and on the (111) surface of single crystals made from palladium ${ }^{32,33,34,35,36}$. On the latter surfaces, the geometric properties 
of moiré patterns induced by graphene ${ }^{32}$ and the electronic structure ${ }^{32,33}$ were subject of studies, as well as the mechanism involved in the graphene growth by carbon segregation ${ }^{36}$.

Although all the latter work gives great insight into graphene on crystalline $\operatorname{Pd}(111)$ surfaces, the PdNP-graphene system needs to be benchmarked by STM, in particular due to the high demand of catalysis ${ }^{14,15}$. Furthermore, although mechanisms of graphene formation by carbon segregation have been proposed ${ }^{36}$, open questions remain still unanswered: in particular, preliminary carbon structures are predicted by theory on $\operatorname{Pd}(111)^{25,26,27}$ but also on other metal surfaces ${ }^{37,38}$. However, the structure of such precursor structures has never been experimentally verified at the atomic scale.

The STM work here shows that during cracking ethylene at high temperatures a carbon precursor structure is formed on the NP's (111) facets prior to graphene formation. Once graphene replaces the latter structure, several moiré patterns with different sizes can be found as well as NP size and shape effects with respect to the moiré patterns and graphene quality. Overall, graphene on PdNPs is different in comparison to graphene on $\operatorname{Pd}(111)$ single crystal surfaces.

\section{Experimental Methods}

Three fresh surfaces are prepared by cleaving HOPG samples in air and cleaned afterwards by annealing at $\sim 650^{\circ} \mathrm{C}$ in a ultra-high vacuum (UHV) chamber during several hours. The PdNPs are then grown by evaporating neutral palladium atoms onto a HOPG sample, which is held at a constant temperature of $480^{\circ} \mathrm{C}$ to guarantee the growth of faceted PdNPs. By varying the deposition time ( 1 to 1.5 minutes), coverages with a nominal thickness between 1 and 1.5 ML are obtained, which results into NPs with a lateral diameter between 10 and $50 \mathrm{~nm}$ and a height between 5 and $10 \mathrm{~nm}$. Due to their 3D shape of a top-truncated 
tetrahedron, the PdNPs exhibit well-known shapes from triangles to regular hexagons via various truncated shapes, with the top facets in a (111) orientation and NP's edges forming angles of $60^{\circ}$ and $120^{\circ 40,47}$. Such as-prepared PdNPs are then annealed at around $\sim 650^{\circ} \mathrm{C}$ in 1 to $2 \times 10^{-6}$ mbar ethylene within max. $\sim 15$ minutes, depending on the chosen dosage. Note that an annealing of as-prepared PdNPs in UHV only $\left(\sim 650{ }^{\circ} \mathrm{C}\right.$, and $\sim 15$ minutes) does not lead to the carbon structures discussed in this work - all carbon precursor and graphene structures on the PdNPs are unambiguously a result of the ethylene annealing and are not due to a possible influence of HOPG on the NP's facets unlike in the case of Ref. ${ }^{20}$. STM experiments are performed in the same UHV chamber ${ }^{48}$ with a commercial standard room temperature AFM/STM and etched tungsten tips. More technical details about the sample preparation and the STM as well as supporting experiments can be found in the Supporting Information.

\section{Results}

Carbon precursor structures. Figure 1 shows STM images obtained after the preparation of PdNPs at $480^{\circ} \mathrm{C}$ in UHV and after a following low ethylene exposure of only $22 \mathrm{~L}(1 \times$ $10^{-6} \mathrm{mbar}_{2} \mathrm{H}_{4}$ ) at around $650^{\circ} \mathrm{C}$. The imaging with atomic resolution is possible with even high tunnel currents $(I)$, low bias voltages $(U)$ and high scanning speeds $(v)$, which is otherwise not possible at as-prepared PdNPs due to tip-induced NP displacements (see Figure S2 in the Supporting Information) - a clear signature that an annealing in ethylene stabilizes the PdNPs, even if the ethylene exposure measures only a few tens of Langmuir. As it will be shown below, carbon structures are formed on the NPs top facets, and the same can be assumed to take place also on the NP's (111) and (100) side facets where such carbon structures are probably linked with the HOPG surface stabilizing the NPs. 
The large-scale image in Figure 1a shows the top (111) facet of a NP with the most important growth structures: a somewhat flat region (1), a thin film partially covering the facet (2a to 2c, 2e and 2f) and some small three-dimensional (3D) islands (3, line profile 1b). On the thin film, a faint hexagonal structure can be seen, which is better resolved in Figure 1c: three more or less regular hexagonal structures exist, which have a dimension of $2.3 \pm 0.2(2 \mathrm{a}), 2.1 \pm 0.2(2 \mathrm{~b})$ and $1.3 \pm 0.2 \mathrm{~nm}(2 \mathrm{~d})$. On other NPs, an even smaller hexagonal structure with a size of $0.72 \pm 0.03 \mathrm{~nm}$ could be found (see Figure S3 in the Supporting Information).

The origin of the hexagonal structures is due to moiré patterns induced by graphene nanosheets on the NP's top facet. This is backed by the height of the film, which can be seen in the profile in Figure 1d: from the lowest part of region 1 in Figure 1c a height of $\sim 2.4 \AA$ is found, which is in good agreement with the height value found for graphene on $\operatorname{Pd}(111)\left(2.3 \AA^{32,39}\right)$.

Because the entire facet is not covered by the film a snapshot of an early growth stage of graphene on PdNPs is presented by Figure 1a and c. The graphene film is composed of three $\sim 15 \mathrm{~nm}$ large islands, which differ by the orientation and size of the moiré patterns (e.g., compare 2a with 2b in Figure 1c), whereas the moiré pattern of an individual island is more or less perfect. The islands show that graphene is formed on the top facets of the NPs, and at the same time graphene also grows at the edges of the (111) facets (e.g., at position $2 \mathrm{e}$ and $2 \mathrm{f}$ in Figure 1a). However, the size of the latter islands is considerably smaller than the one of the islands on the facet, which obviously means that the growth is faster on the facet than at the edges.

Interestingly, in region 1 of Figure 1c an irregular structure can be found, which covers the entire flat region 1 in Figure 1a. Figure 1e shows the same irregular structure on another 

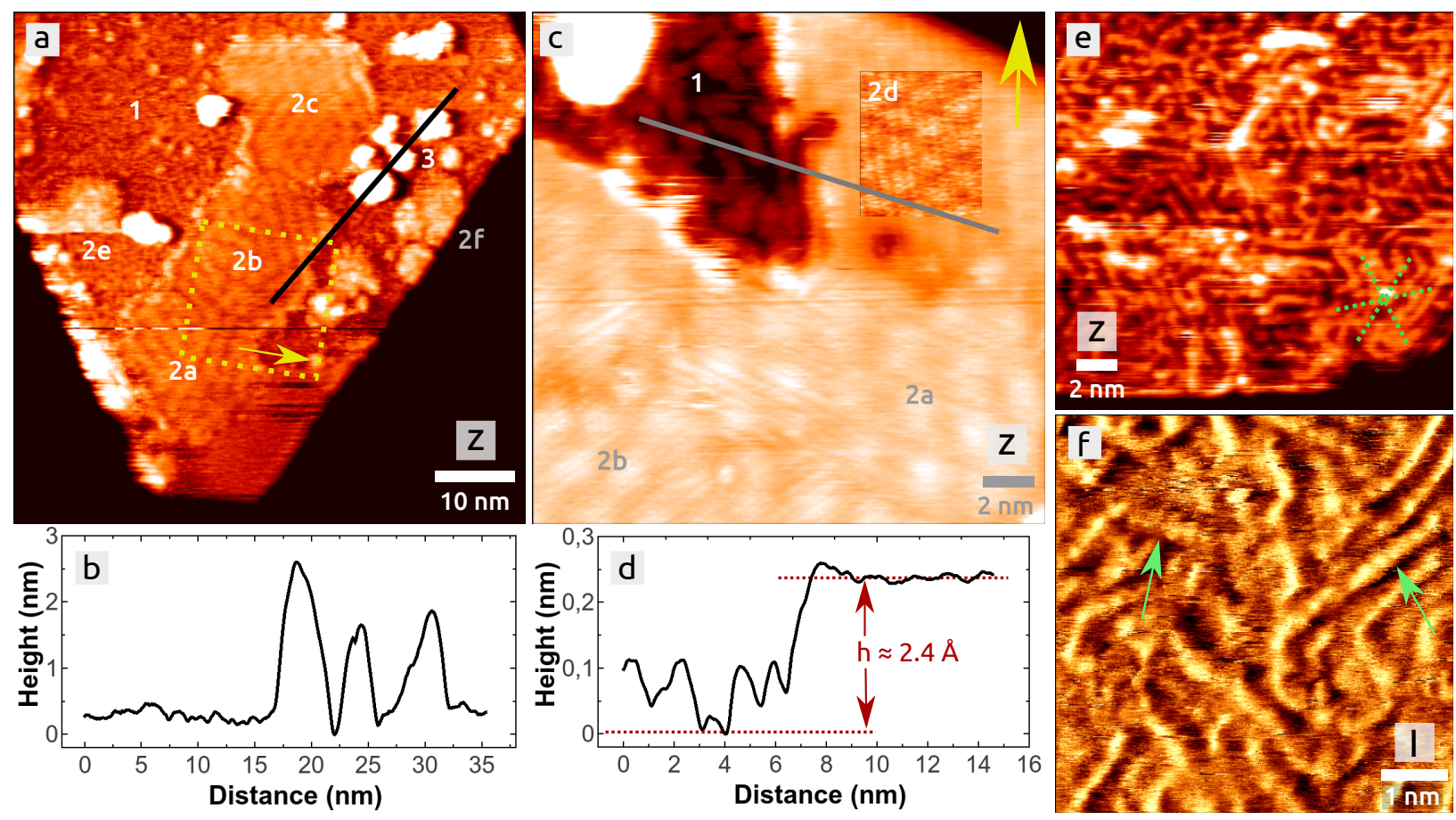

Figure 1: The (111) top facet of PdNPs after an annealing in $22 \mathrm{~L}$ of $\mathrm{C}_{2} \mathrm{H}_{4}$ at $665{ }^{\circ} \mathrm{C}$. (a) Large-scale topography STM image and corresponding line profile (b) presenting important structural features on the facet discussed in the main article $(U=1.83 \mathrm{~V} ; I=0.15 \mathrm{nA}$; $v=3.3 \mathrm{~Hz}$ ). (c) A successive topography image with profile (d) presenting different graphene moiré patterns (2a, 2b, 2c) and the filament structure $(1)(U=0.20 \mathrm{~V} ; I=1.37 \mathrm{nA} ; v=2.4$ $\mathrm{Hz}$ ). The image shows the region marked by the yellow dotted square in image (a) (image orientation: compare yellow arrows) whereas the inset shows an increased contrast of the image. (e) Topography image of the filament structure on the top (111) facet of another $\mathrm{PdNP}$, after an post-annealing of the same sample at $673^{\circ} \mathrm{C}$ for $1 \mathrm{~h}$ in $\mathrm{UHV}(U=0.25 \mathrm{~V}$; $I=0.25 \mathrm{nA} ; v=3.9 \mathrm{~Hz}$ ). (f) An atomically resolved image of possible carbon chains (green arrows). The image shows the residual tunnel current of the topography image in Figure S2 of the Supporting Information $(U=0.027 \mathrm{~V} ; I=2.11 \mathrm{nA} ; v=5.0 \mathrm{~Hz})$.

NP with a higher resolution: the irregular structure is composed by a dense arrangement of a few nanometer long and 3 to $4 \AA$ thin filaments, which have a height of around $1 \AA$ (see Figure S4 and S5 in the Supporting Information). Some filaments are curved whereas others are straight being roughly oriented along three surface directions forming angles of 60 and $120^{\circ}$ (see green cross in Figure 1e). Figure 1f shows the same NP facet and also 
the filament structure partially with atomic resolution. It seems that a filament is formed by a chain of carbon atoms, which keep a mean distance of $2.5 \pm 0.3 \AA$ to each other. For an unambiguous identification of such a structure a comparison is needed between density functional theory (DFT) as accomplished in, e.g., Ref. ${ }^{27}$ and experiments, which should be preferably conducted at liquid helium temperature to increase the lateral STM resolution.

The entire filament structure is in perfect agreement with the current picture of carbon on and inside palladium (111): during the ethylene exposure at $665{ }^{\circ} \mathrm{C}$ ethylene completely decomposes to molecular hydrogen, which leaves the NPs, and to carbon that is left at the $\mathrm{NPs}^{24}$. In the very initial steps of the carbon contamination, the first individual carbon atoms move below the surface as shown by density functional theory (DFT) on $\operatorname{Pd}(111)$ surfaces ${ }^{23,25,26,28}$ and on the facets of $\operatorname{PdNPs}^{22}$, being in agreement with experiments on $\operatorname{Pd}(111)^{21,24}$ and with $\operatorname{PdNPs}^{29}$. The DFT studies show that upon an increasing amount of carbon it is less probable for carbon to go subsurface $25,26,27,28$, starting with a relative carbon content of $\sim 0.5$ (maximum coverage of, e.g., the three-fold fcc hollow sites) ${ }^{25}$. At a content of $\sim 1$ the most stable configurations involve carbon on the surface and in the subsurface $^{25,26}$. Interestingly, chains of carbon atoms are predicted, which may form a CPd-C bridging-metal structure at the beginning ${ }^{27}$ and carbon oligomers including more than 3 carbon atoms ${ }^{25,27}$. The mean C-C distance in an oligomer measures about $\sim 1.3 \AA^{25}$ whereas the carbon atoms exhibit a rough vertical zigzag shape, with the central carbon atom on the top of a palladium atom and adjacent carbon atoms in hollow sites on $\mathrm{Pd}(111)$. If it is assumed that the filaments in Figure 1f are indeed carbon chains it can be speculated that because of the vertical zigzag shape of the carbon atoms from above only each second carbon atom is imaged by STM, which explains the distance of $2.5 \AA$.

Overall, the filament structure shown in Figure 1 is a preliminary carbon precursor struc- 
ture before the formation of graphene starts, and it is composed of surface and possibly also subsurface carbon. As shown in the following, graphene replaces the filament structure and the 3D islands when more carbon reaches the NP's facets upon higher ethylene exposures.

Graphene moiré patterns. After a high dosage of ethylene ( $\gg 100 \mathrm{~L}$ ) at $\sim 650{ }^{\circ} \mathrm{C}$, graphene is formed on the NP's facets. Figure 2 shows a collection of five G@PdNPs obtained from five individual STM measurements. The top (111) facet of NP I has a lateral diameter of $\varnothing=35 \mathrm{~nm}$ whereas the size decreases for all other NPs down to a diameter of $\varnothing=10 \mathrm{~nm}$ for NP V. Because the properties of graphene (geometry and contrast of moiré patterns, atomic structure, etc.) discussed in the entire work do not depend on the ethylene exposure, it is anticipated that graphene grows as a single sheet only, which is strongly supported by work done on the $\operatorname{Pd}(111)$ single crystal surfaces ${ }^{32}$.

For NPs with a lateral size larger than $\varnothing \approx 30 \mathrm{~nm}$, the first important characteristic of graphene is that in many cases only one moiré pattern exists, which covers the entire top (111) facet with almost no imperfections in the moiré lattice. The moiré pattern is as perfect as exemplified by NP I whereas on other NPs a few defects can be observed, which, however, do not strongly disturb the moiré pattern (see Figure S6 in the Supporting Information). The second important characteristic is that the dimension of a single moiré pattern is always larger than $\sim 2 \mathrm{~nm}$, e.g., the moiré pattern of NP I has a dimension of $2.6 \pm 0.1 \mathrm{~nm}$. Under the assumption that the epitaxy between graphene and the palladium (111) facet is commensurate $^{32,33}$ by an adjustment of only the graphene lattice (lattice contraction/expansion $\rho+$ rotation by $\alpha_{\mathrm{Pd}-\mathrm{G}}$ formed between the palladium $[1 \overline{1} 0]_{\mathrm{Pd}}$ and graphene $[2 \overline{1} \overline{1} 0]_{\mathrm{G}}$ direction) a precise description of the moiré's unit cell can be obtained from only the experimentally obtained length of the unit cell vector $\hat{\lambda}_{\mathrm{M}}$ and the angle $\hat{\alpha}_{\mathrm{Pd}-\mathrm{M}}$ between the latter vector and the edges of the NP's facets (see Figure S1 in the Supporting Information). Here, it is 


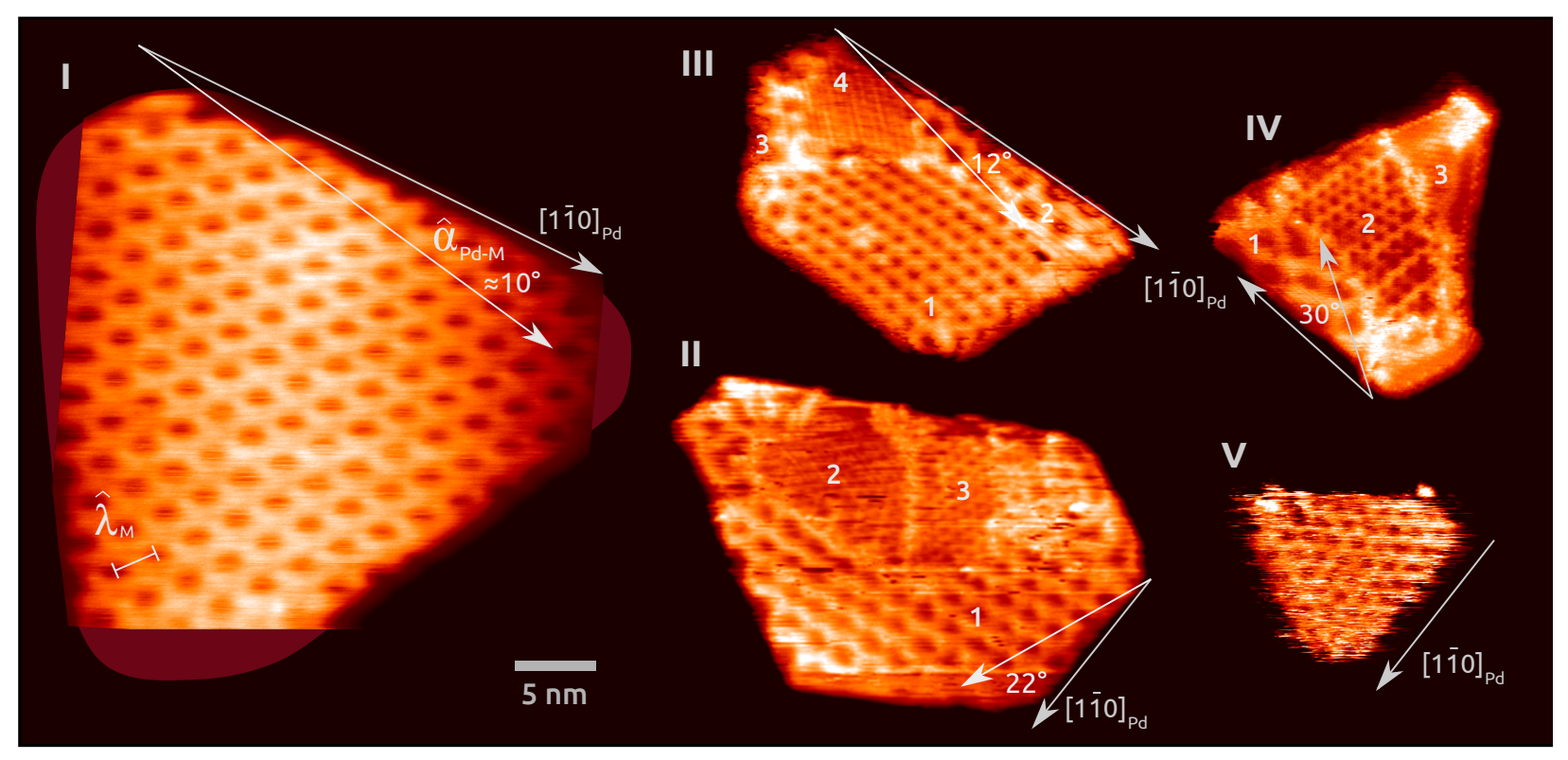

Figure 2: Different graphene moiré patterns observed by STM on the top (111) facets of five selected G@PdNPs (I-V) after a high exposure of $\mathrm{C}_{2} \mathrm{H}_{4}$ at $665^{\circ} \mathrm{C}$ (NP I: $847 \mathrm{~L}, \mathrm{NP}$ II, III and IV: $2835 \mathrm{~L}, \mathrm{NP}$ V: $585 \mathrm{~L}$ ). Each NP was imaged in an individual STM measurement whereas its image was cut and assembled into the Figure with the slow scanning direction kept in a horizontal orientation. Because of the different measurement conditions (different scanning angles, different samples, etc.) the five NPs exhibit arbitrarily formed angles to each other. All images show the topography signal. The artificial dark red drawing below NP I shall symbolize the NP's shape obtained from other large-scale, low-resolution STM images (not shown). Scanning parameters: $U=0.41 \mathrm{~V} ; I=0.43 \mathrm{nA} ; v=3.3 \mathrm{~Hz}(\mathrm{NP}$ I), $U=0.20 \mathrm{~V} ; I=1.04 \mathrm{nA} ; v=2.0 \mathrm{~Hz}(\mathrm{NP}$ II), $U=-0.077 \mathrm{~V} ; I=1.71 \mathrm{nA} ; v=1.2 \mathrm{~Hz}(\mathrm{NP}$ III), $U=0.14 \mathrm{~V} ; I=1.47 \mathrm{nA} ; v=5.0 \mathrm{~Hz}(\mathrm{NP}$ IV), $U=0.025 \mathrm{~V} ; I=0.85 \mathrm{nA} ; v=3.0 \mathrm{~Hz}$ $(\mathrm{NP} V)$.

assumed that the NP's edges run along equivalent $\left\langle 1 \overline{1} 0>_{\text {Pd }}\right.$ surface directions on the palla$\operatorname{dium}$ (111) facets as verified before ${ }^{40}$. With the experimental values of $\hat{\lambda}_{\mathrm{M}}=2.6 \pm 0.1 \mathrm{~nm}$ and $\hat{\alpha}_{\mathrm{Pd}-\mathrm{M}}=10 \pm 1^{\circ}$ for NP I, a $\lambda_{\mathrm{M}}=2.52 \mathrm{~nm}$ large $(2 \sqrt{21} \times 2 \sqrt{21})-R 10.9^{\circ}$ unit cell is found, which is created by a rotation of $\alpha_{\mathrm{Pd}-\mathrm{G}}=+1.1^{\circ}$ and an expansion of $\rho=0.83 \%$ of the free-standing graphene lattice (see ball model in Figure 3e). This moiré pattern is similar in size with others, e.g., a $\lambda_{\mathrm{M}}=2.47 \mathrm{~nm}$ large $(9 \times 9)-R 0.0^{\circ}$ unit cell can be frequently observed (see Figure $\mathrm{S} 6$ in the Supporting Information), where both angles, $\alpha_{\mathrm{Pd}-\mathrm{M}}$ and $\alpha_{\mathrm{Pd-G}}$, 

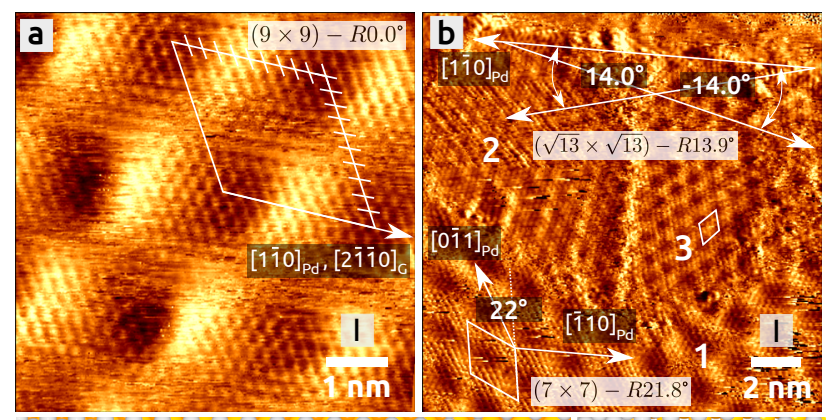

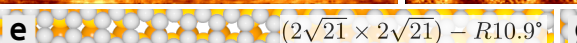

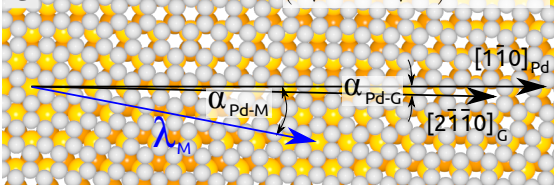

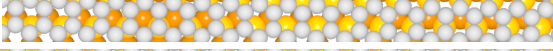

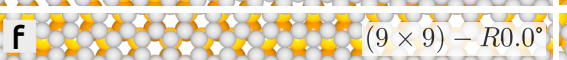

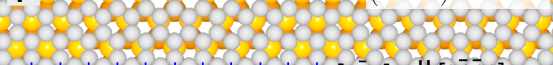

$11+1,1+1-[110]_{P d} \|[2110]_{G}$

$0080 \lambda_{M}$

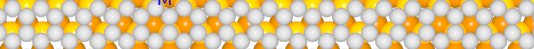

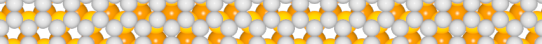

C $\bigcirc$ Surface Pd $\bigcirc$ Subsurf. Pd $\odot$

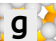

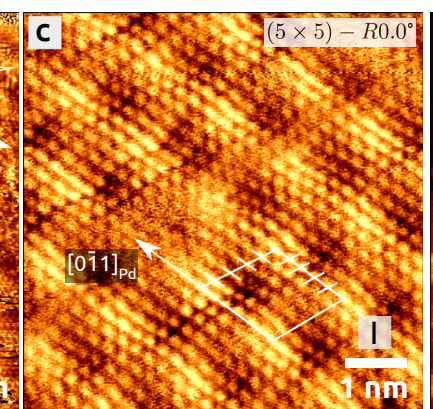
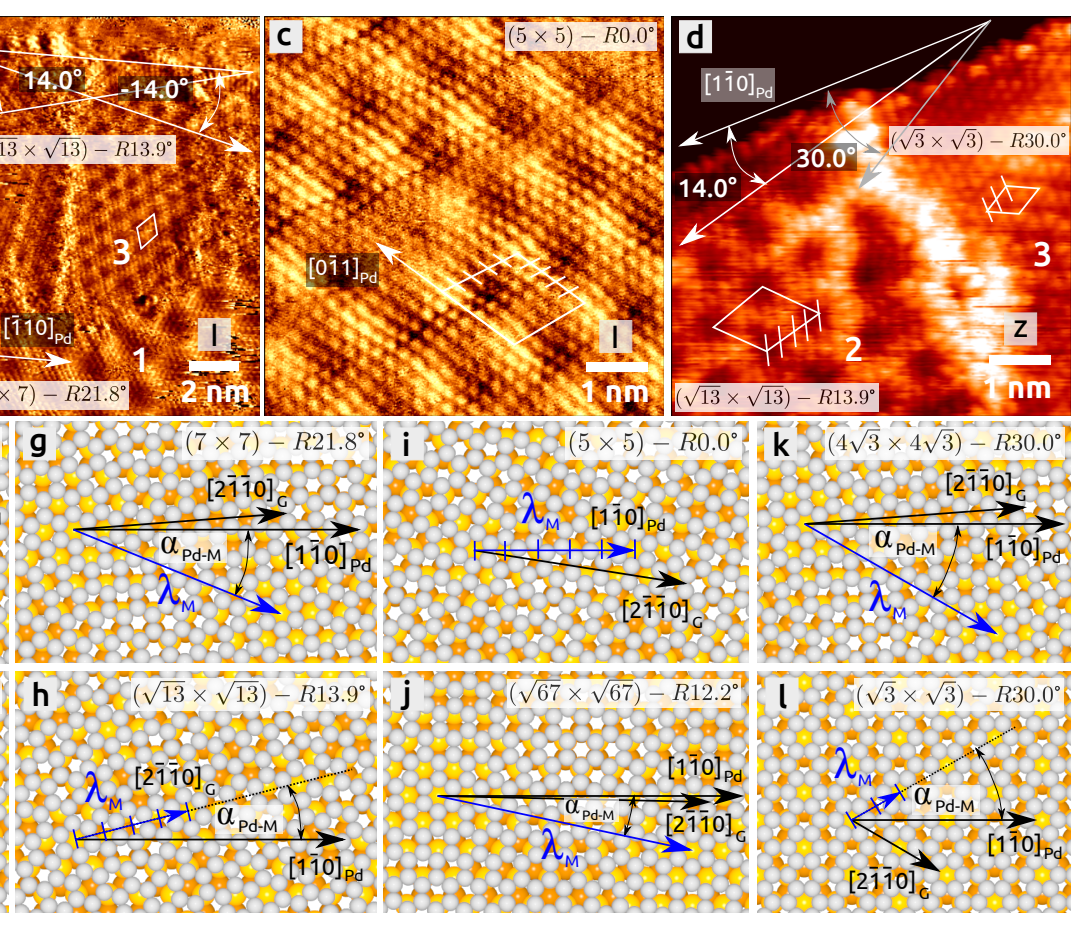

Figure 3: The atomic structure of some selected moiré patterns imaged by STM (a-d) and models for most of the moiré patterns observed in this work (e-l). Images (a-c) represent the tunnel current signal whereas image (d) shows the topography signal. Image (a) is from the NP in the Supporting Information (Figure S5b) and images (b-d) are from NP II (1), III(1) and IV (Figure 2), respectively. Scanning parameters: $U=0.056 \mathrm{~V} ; I=1.04 \mathrm{nA} ; v=5.6$ $\mathrm{Hz}(\mathrm{a}), U=0.2 \mathrm{~V} ; I=1.04 \mathrm{nA} ; v=4.9 \mathrm{~Hz}(\mathrm{~b}), U=-0.077 \mathrm{~V} ; I=1.71 \mathrm{nA} ; v=6.7 \mathrm{~Hz}$ (c), $U=0.14 \mathrm{~V} ; I=1.47 \mathrm{nA} ; v=5.4 \mathrm{~Hz}$ (d). In the models (e-l) most important details are shown whereas Figure S1 in the Supporting Information documents all relevant vectors, distances and angles.

are zero (see ball model in Figure 3f). The analysis is more precise if the atomic resolution is obtained: the atomically resolved image in Figure 3 a shows a $(9 \times 9)-R 0.0^{\circ}$ moiré pattern found on the NP in the Supporting Information (Figure S6b). The unit cell vector of the moiré pattern is spanned by 9 sections of $10 \mathrm{Pd}$ atoms and in particular 10 sections of 11 graphene units (6-membered hexagonal ring) corresponding to the 10 atomic sections found in the experimental image (compare dark blue sections in Figure 3f with bright sections in 
Figure 3a).

When comparing large G@PdNPs with NPs of smaller size (e.g., NP I with NPs II, III and IV in Figure 2) an interesting difference can be seen: for almost all NPs smaller than $\varnothing \approx 25 \mathrm{~nm}$, graphene does not exhibit anymore only one perfect moiré pattern covering the entire (111) facet of a NP: at least two graphene regions can be observed either forming two domains of moiré patterns with identical sizes or moiré patterns with different sizes. For instance, three distinct moiré patterns can be seen on NP II, with the largest located in region 1. With the experimental values listed in Table 1, the same type of analysis from above yields a $\lambda_{\mathrm{M}}=1.92 \mathrm{~nm}$ large $(7 \times 7)-R 21.8^{\circ}$ unit cell for the latter moiré pattern (see model in Figure 3g). Figure 3b shows the top (111) facet of NP II in a higher resolution. Interestingly, the atomic rows in region 1 run along equivalent palladium $<1 \overline{1} 0>_{\mathrm{Pd}}$ directions, which indicates that the Pd atoms of the (111) facet contribute to the atomic contrast (see Figure S7 in the Supporting Information). In comparison to the moiré pattern 1, the moiré patterns 2 and 3 are much smaller but they are equal in size. They only differ by their rotation with respect to the NP's $[1 \overline{1} 0]_{\mathrm{Pd}}$ edge $\left(\hat{\alpha}_{\mathrm{Pd}-\mathrm{M}}=+14 \pm 1^{\circ}(2), \hat{\alpha}_{\mathrm{Pd}-\mathrm{M}}=-14 \pm 1^{\circ}(3)\right)$, therefore forming two domains of one type of moiré pattern. They have a $(\sqrt{13} \times \sqrt{13})-R 13.9^{\circ}$ unit cell with a length of $\lambda_{\mathrm{M}}=0.90 \mathrm{~nm}$ as shown by the model in Figure $3 \mathrm{~h}$.

Several moiré patterns can be observed also at NPs III and IV (Figure 2): NP III has three moiré patterns with three different sizes. The orientation of the moiré pattern in region 1 is parallel to the NP's edges and run therefore along the equivalent palladium $<1 \overline{1} 0>_{\mathrm{Pd}}$ directions. Because of its small size $\left(\hat{\lambda}_{\mathrm{M}}=1.4 \pm 0.1\right)$ and the 5 atomic sections, which are formed by $6 \mathrm{Pd}$ atoms and span the length of the unit cell's vector (see white sections in the atomically resolved image in Figure 3c and compare with Figure 3i), the moiré pattern must have a $(5 \times 5)-R 0.0^{\circ}$ unit cell with a length of $\lambda_{\mathrm{M}}=1.37 \mathrm{~nm}$. Interestingly, in region 2 and 
Table 1: All moiré patterns observed so far on G@PdNPs. The parameters $\hat{\lambda}_{M}$ and $\hat{\alpha}_{\mathbf{P d}-\mathrm{M}}$ are the experimental values for the length of the moiré's unit cell vector and the angle between the unit cell vector and the $\langle 1 \overline{1} 0\rangle_{\mathbf{P d}}$ direction whereas $\lambda_{\mathrm{M}}$ and $\alpha_{\mathrm{Pd}-\mathrm{G}}$ are same parameters resulting from the model for the moiré pattern. The parameter $\rho=a_{\mathbf{G}}-a_{\mathbf{G}}$ free $/ a_{\mathbf{G}}$ free denotes the contraction/expansion factor of the graphene lattice ( $\operatorname{siz} \bar{e} a_{\mathrm{G}}$ ) with respect to free-standing graphene, which has a size of $a_{\mathrm{G} \text { free }}=2.463 \AA^{41,42}$. For $\mathrm{Pd}(111)$, a unit cell length of $a_{\mathbf{P d}}=3.889 \AA$ is used $^{43, \overline{4}, 45}$, which results into a next-neighbor distance of two Pd atoms of $d_{\mathbf{P d}-\mathrm{Pd}}=2.750 \AA$. The abbreviation MB stands for moiré pattern whereas the number is the one of the moiré pattern on the respective NP.

\begin{tabular}{|lllllll|}
\hline$\hat{\lambda}_{\mathrm{M}}(\mathrm{nm})$ & $\hat{\alpha}_{\mathrm{Pd}-\mathrm{M}}\left(^{\circ}\right)$ & Unit cell & $\lambda_{\mathrm{M}}(\mathrm{nm})$ & $\rho(\%)$ & $\alpha_{\text {Pd-G }}$ & Figure \\
\hline \hline $0.5 \pm 0.1$ & $30 \pm 1$ & $(\sqrt{3} \times \sqrt{3})-R 30.0^{\circ}$ & 0.48 & -3.3 & 30.0 & $\begin{array}{l}\text { Fig. 2: NP IV (MP 3) } \\
\text { Fig. 3d (MP 3) }\end{array}$ \\
& & & & & & Fig. S3 \\
\hline $0.72 \pm 0.03$ & $19 \pm 1$ & $(\sqrt{7} \times \sqrt{7})-R 19.1^{\circ}$ & 0.73 & -1.5 & 19.1 & Fig. 2: NP IV (MP 4) \\
\hline $0.9 \pm 0.1$ & $14 \pm 1$ & $(\sqrt{13} \times \sqrt{13})-R 13.9^{\circ}$ & 0.99 & +0.6 & 13.9 & $\begin{array}{l}\text { Fig. 2: NP II (MP 2, 3) } \\
\end{array}$ \\
& & & & & Fig. 3 (MP 4) \\
\hline $1.4 \pm 0.1$ & $0 \pm 1$ & $(5 \times 5)-R 0.0^{\circ}$ & 1.37 & +0.3 & -8.9 & Fig. 1c (2d) \\
& & & & & Fig. 2: NP III (MP 1) \\
& & & & & Fig. 2: NP V \\
\hline $2.0 \pm 0.1$ & $30 \pm 1$ & $(4 \sqrt{3} \times 4 \sqrt{3})-R 30.0^{\circ}$ & 1.91 & -1.0 & -3.7 & Fig. 2: NP IV (MP 1) \\
\hline $2.0 \pm 0.1$ & $22 \pm 1$ & $(7 \times 7)-R 21.8^{\circ}$ & 1.92 & +0.1 & +4.5 & Fig. 2: NP II (MP 1) \\
\hline $2.2 \pm 0.2$ & $12 \pm 2$ & $(\sqrt{67} \times \sqrt{67})-R 12.2^{\circ}$ & 2.25 & $\sim 0.0$ & +0.6 & Fig. 1c (2b) \\
& & & & & & Fig. 2: NP III (MP 2, 3) \\
\hline $2.5 \pm 0.1$ & $0 \pm 1$ & $(9 \times 9)-R 0.0^{\circ}$ & 2.47 & +0.5 & 0.0 & Fig. 1c (2a) \\
& & & & & Fig. 3a \\
\hline $2.6 \pm 0.1$ & $10 \pm 1$ & $(2 \sqrt{21} \times 2 \sqrt{21})-R 10.9^{\circ}$ & 2.52 & +0.83 & 1.1 & Fig. 2: NPI \\
\hline
\end{tabular}

3 (Figure 2) relatively large moiré patterns can be seen at the edges of the NPs covering only a small region of the NP's facet. They have the same size and form two domains, having possibly a $(\sqrt{67} \times \sqrt{67})-R 12.2^{\circ}$ unit cell with a length of $\lambda_{\mathrm{M}}=2.25 \mathrm{~nm}$ (see ball model in Figure 3j). In contrast to all other patterns, the last pattern in region 4 has no clear definition of a moiré pattern.

Similar to NP III, three moiré patterns can also be found for NP IV (Figure 2): possibly 
$\mathrm{a}(4 \sqrt{3} \times 4 \sqrt{3})-R 30.0^{\circ}\left(\lambda_{\mathrm{M}}=1.91 \mathrm{~nm}\right)$ moiré pattern in region 1 at the edge of the NP, a smaller $(\sqrt{13} \times \sqrt{13})-R 13.9^{\circ}\left(\lambda_{\mathrm{M}}=0.99 \mathrm{~nm}\right)$ moiré pattern in the center of the NP (region 2 ) and the smallest $(\sqrt{3} \times \sqrt{3})-R 30.0^{\circ}\left(\lambda_{\mathrm{M}}=0.48 \mathrm{~nm}\right)$ moiré pattern in region 3 . The first $4 \sqrt{3} \times 4 \sqrt{3}$ moiré pattern (see ball model in Figure 3k) is yet another large pattern covering only a small fraction of the NP's facet at the edge whereas the $\sqrt{13} \times \sqrt{13}$ moiré pattern is the same as the one found on NP II. As it can be seen in the atomically resolved image in Figure 3d, 4 atomic sections span the unit cell of this moiré pattern, which corresponds to 4 sections formed by 5 graphene units (see dark blue sections in Figure 3h). Quite similar, the length of the very small $\sqrt{3} \times \sqrt{3}$ unit cell in region 3 is formed by 2 atomic sections, which would correspond to 2 sections formed by 3 graphene units (see dark blue sections in Figure 31).

\section{Discussion}

Apart from interesting findings with respect to the atomic contrast (see Figures S7 and S8 in the Supporting Information), the most fascinating result of the entire work on G@PdNPs is that several moiré patterns exist on preferentially small NPs, which is also the case if the growth temperature is at $730^{\circ} \mathrm{C}$, as verified by recent experiments (not shown). This automatically implies that graphene is not growing perfectly in one single, defect-free sheet on a (111) facet as in the case of large NPs. It is rather present in several nanosheets, which are rotated to each other producing different moiré patterns as observed by STM. With this, several defects along the borders of the graphene nanosheets exist, as it can be seen in Figure 2 and Figure $3 \mathrm{~b}$ and $\mathrm{d}$.

An astonishing result is the occurrence of 9 moiré patterns that can be observed on the NPs listed in Table 1 and on many other NPs (not shown). These are 3 patterns more than 
observed on the $\operatorname{Pd}(111)$ single-crystal surface ${ }^{33}$ whereas only 2 moiré patterns on $\operatorname{Pd}(111)$ are also observed on the PdNPs, namely the $0.73 \mathrm{~nm}$ small $(\sqrt{7} \times \sqrt{7})-R 19.1^{\circ}$ and the $0.99 \mathrm{~nm}$ small $(\sqrt{13} \times \sqrt{13})-R 13.9^{\circ}$ moiré pattern. All the other moiré patterns on the $\operatorname{Pd}(111)$ single-crystal surface $\left((3 \sqrt{7} \times 3 \sqrt{7})-R 19.1^{\circ},(\sqrt{39} \times \sqrt{39})-R 16^{\circ},(\sqrt{21} \times \sqrt{21})-R 11^{\circ}\right.$ and $\left.(7 \times 7)-R 0^{\circ}\right)^{33}$ have not been observed on G@PdNPs so far. From the current statistics of $20 \mathrm{NPs}$ it seems that the growth of graphene on the PdNPs is different with respect to $\operatorname{Pd}(111)$. The reason for this might be the clamping of graphene by surface steps on the $\operatorname{Pd}(111)$ surface because graphene is embedded into the $\operatorname{Pd}(111)$ surface $^{32}$. This may lead to different growth conditions hindering a 'free orientation' of graphene on $\mathrm{Pd}(111)$ during the growth, unlike the case on the PdNPs, where graphene is not hindered by steps.

Another observation is that the values of the expansion/compression factor (see Table 1) are all mostly around or even below 1\% (Table 1), similar to values observed on many other metal surfaces ${ }^{17}$. A clear exception is the smallest of all patterns, namely the $(\sqrt{3} \times$ $\sqrt{3})-R 30.0^{\circ}$ moiré pattern with its $3.3 \%$ lattice contraction. Interestingly, it has the unit cell dimension of the most stable carbide phase on the (111) surface of $\mathrm{Pd}_{6} \mathrm{C}^{28}$, which allows speculating that the graphene layer is stabilized by $\mathrm{Pd}_{6} \mathrm{C}$ underneath.

The fundamental question is, why several moiré patterns appear on small NPs, which contrasts the single large moiré patterns that appear on many large NPs $(\varnothing>30 \mathrm{~nm})$. The second question with respect to the small NPs is why very small and large moiré patterns appear on one and the same top (111) facet being densely packed on a small surface area. This contrasts the situation on the $\mathrm{Pd}(111)$ single-crystal surface where graphene produces much more homogeneous moiré patterns, if not only one pattern, on terraces of similar size ${ }^{32}$. As pointed out before, graphene starts to grow in particular on the (111) facets, creating islands of nanographene with a size of $\varnothing \approx 15 \mathrm{~nm}$ (Figure 1a), which seems to be close to 
the critical island size where graphene sheets coalesce. For large NPs it can be assumed that the graphene formation is exactly the same for all nucleation sites on the top (111) facets ${ }^{46}$, where the Pd lattice is almost defect-free. This results into one almost defect-free graphene sheet and in turn into a uniform and perfect moiré pattern on the entire large NP. In the case of small NPs there is a clear competition between probably only one nucleation site on the (111) top facet and in particular several nucleation sites at the edges of the facet. It is evident that the edges of the NPs, probably in conjunction with the side facets of the NPs, determine the orientation of the graphene at the edges. As it can be seen in Figure $3 \mathrm{~d}$ (NP IV) or in Figure 2 (NP II and III), the edges are not throughout perfectly straight at the atomic scale although they form overall perfect $60^{\circ}$ and $120^{\circ}$ angles of the top (111) facet at the nanometer scale. These local imperfections of the edges are due to kinks at the edges or small sections of edges, which shortly change the direction of the otherwise ideal $<1 \overline{1} 0>_{\text {Pd }}$ edge. They can be possibly created during the growth of the NPs or during the growth of graphene, as shown on the $\mathrm{Pd}(111)$ single crystal surface where step forming $\mathrm{Pd}$ atoms are mobile during the growth ${ }^{32}$. Such local imperfections at the NP's edges could then lead to different moiré patterns at the edges. A direct proof are highly symmetric NPs with perfect edges along equivalent $\left\langle 1 \overline{1} 0>_{\text {Pd }}\right.$ directions where the graphene must be perfect on the top (111) facet. Indeed, this can be observed in rare cases, as shown by NP V in Figure 2: the NP exhibits perfectly straight edges along equivalent $\left\langle 1 \overline{1} 0>_{\mathrm{Pd}}\right.$ directions but also a perfect epitaxy with the HOPG substrate (see Figure 4). As a consequence, the single moiré pattern with its $(5 \times 5)-R 0.0^{\circ}$ unit cell is uniform and defect-free. 


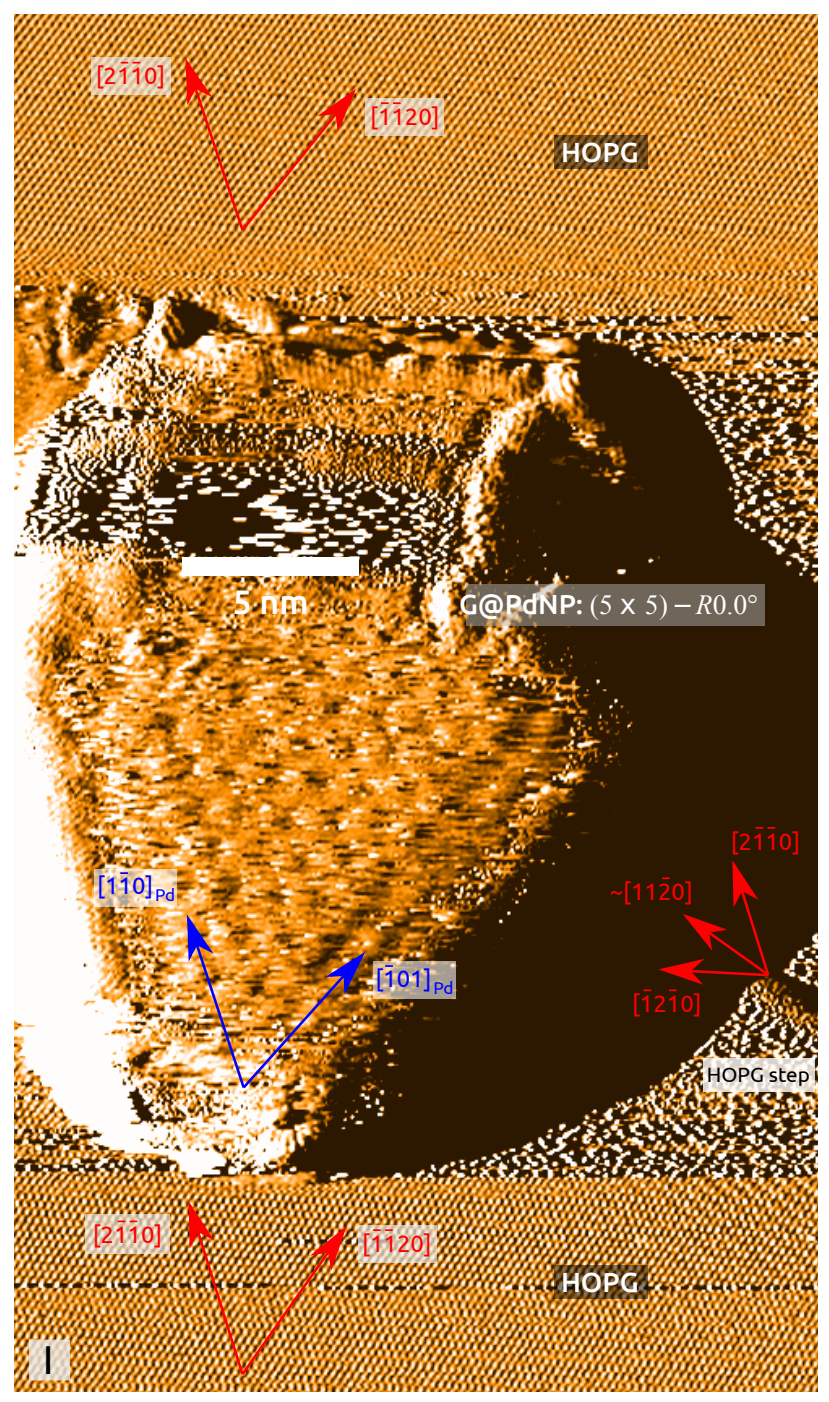

Figure 4: The epitaxy of NP V from Figure 2 on the HOPG surface. Thanks to the small lateral size of $11.4 \mathrm{~nm}$ and the relatively small height of $4.7 \mathrm{~nm}$, the atomic resolution could be obtained on the HOPG substrate surface while imaging the $1.37 \mathrm{~nm}$ large $(5 \times 5)-R 0.0^{\circ}$ moiré pattern. The NP's equivalent $\langle 1 \overline{1} 0\rangle_{\text {Pd }}$ edges are oriented parallel to equivalent $\left.<2 \overline{1} 10\right\rangle$ directions of the HOPG surface. Furthermore, they form perfect $60^{\circ}$ angles so that the NP exhibits a highly symmetric shape. The NP is attached at a step, which probably runs along a $<11 \overline{2} 0>$ direction. $\left(U_{\text {Bias }}=0.015 \mathrm{~V}, I=1.47 \mathrm{nA}, v=3.0 \mathrm{~Hz}\right)$. 


\section{Conclusion}

STM measurements on HOPG supported PdNPs show that by thermal decomposition of ethylene at $650^{\circ} \mathrm{C}$ a precursor structure is formed first on the (111) facets prior to graphene formation. The precursor structure is composed of carbon, which forms nanometer long filaments on the entire facet. Once this structure is replaced by single-layer graphene upon dosing the NPs with a high ethylene dosage, up to 9 different graphene moiré patterns can be found on the NPs, from which only 2 coincide with 6 moiré patterns found on crystalline metal $\operatorname{Pd}(111)$ surfaces $^{33}$. This already is a signature that graphene on PdNPs is different in comparison with its bulk counterpart, which might be the case also for other NPs like Ru, Rh, $\mathrm{Co}, \mathrm{Fe}, \mathrm{Cu}$ or PtNPs, and NPs composed of two different elements like bi-metallic NPs.

The work delivers the clear and important message that below a specific NP size $(\approx 30 \mathrm{~nm}$ for PdNPs) graphene does not grow in a single perfect sheet but is present in the form of several nanosheets exhibiting relatively small and large moiré patterns densely packed on a small surface area, which contrasts the situation on the $\operatorname{Pd}(111)$ single-crystal surface where more homogeneous moiré patterns are found. Most importantly, several line defects can be observed in graphene on the NP's facets whereas defects probably also exist at the NP's edges. Only highly symmetric, well-faceted NPs lead to one perfect graphene layer on a facet. The various line defects created by several nanographene sheets can be active catalytic centers whereas for catalysis under cover ${ }^{15}$ they are probably the principle gate for basic reactant and product molecules, which can access the nanocontainer formed by graphene and the NP's facets via the defects. Because of the general importance of those NPs, which are encapsulated by doped graphene ${ }^{6,7,9}$, the work motivates to study same NPs after a doping of graphene with, e.g., nitrogen, which can be principally accomplished by post-synthesis treatments like gentle $\mathrm{N}_{2}^{+}$ion bombardment ${ }^{49}$, post-annealing in ammonia ${ }^{50}$ 
or by using a nitrogen plasma ${ }^{51}$.

\section{Associated Content}

\section{Supporting Information}

The Supporting Information is available free of charge on the ACS Publications website at DOI: ... . Details about the sample preparation, STM measurements, image analysis and supporting experiments (PDF).

\section{Author information}

\section{Corresponding author}

E-mail: barth@cinam.univ-mrs.fr

\section{ORCID}

Clemens Barth: 0000-0003-4250-4533

\section{Author contributions}

The entire experimental work, image analysis, data interpretation, conclusion and the writing of the manuscript was done by C. B. alone.

\section{Notes}

The author declares no competing financial interests.

\section{Acknowledgements}

The author highly acknowledges G. Arthaud and C. Coudreau for maintenance work at the UHV chamber and A. Ranguis for the experimental procedure needed to prepare STM tips. He also expresses his very great appreciation to H. Grönbeck for very stimulating discussions. The author declares no competing financial interest. 


\section{References}

(1) Argyle, M.; Bartholomew, C. Heterogeneous Catalyst Deactivation and Regeneration: A Review. Catalysts 2015, 5, 145-269.

(2) Teschner, D.; Borsodi, J.; Wootsch, A.; Revay, Z.; Havecker, M.; Knop-Gericke, A.; Jackson, S. D.; Schlögl, R. The Roles of Subsurface Carbon and Hydrogen in PalladiumCatalyzed Alkyne Hydrogenation. Science 2008, 320, 86-89.

(3) Freund, H.-J. Model Studies in Heterogeneous Catalysis. Chemistry-A European Journal 2010, 16, 9384-9397.

(4) De Smit, E.; Weckhuysen, B. M. The Renaissance of Iron-Based Fischer-Tropsch Synthesis: on the Multifaceted Catalyst Deactivation Behaviour. Chem. Soc. Rev. 2008, 37, 2758 .

(5) Diarra, M.; Zappelli, A.; Amara, H.; Ducastelle, F.; Bichara, C. Importance of Carbon Solubility and Wetting Properties of Nickel Nanoparticles for Single Wall Nanotube Growth. Phys. Rev. Lett. 2012, 109, 2758.

(6) Hu, L.; Zhang, R.; Wei, L.; Zhang, F.; Chen, Q. Synthesis of FeCo Nanocrystals Encapsulated in Nitrogen-Doped Graphene Layers for Use as Highly Efficient Catalysts for Reduction Reactions. Nanoscale 2015, 7, 450-454.

(7) Kim, H.; Robertson, A. W.; Kim, S. O.; Kim, J. M.; Warner, J. H. Resilient High Catalytic Performance of Platinum Nanocatalysts with Porous Graphene Envelope. ACS Nano 2015, 9, 5947-5957.

(8) Zeng, M.; Liu, Y.; Zhao, F.; Nie, K.; Han, N.; Wang, X.; Huang, W.; Song, X.; Zhong, J.; Li, Y. Metallic Cobalt Nanoparticles Encapsulated in Nitrogen-Enriched Graphene 
Shells: Its Bifunctional Electrocatalysis and Application in Zinc-Air Batteries. Adv. Funct. Mater. 2016, 26, 4397-4404.

(9) Xu, Y.; Tu, W.; Zhang, B.; Yin, S.; Huang, Y.; Kraft, M.; Xu, R. Nickel Nanoparticles Encapsulated in Few-Layer Nitrogen-Doped Graphene Derived from Metal-Organic Frameworks as Efficient Bifunctional Electrocatalysts for Overall Water Splitting. Adv. Mater. 2017, 29, 1-8.

(10) Ren, X.; Zi, W.; Wei, Q.; Liu, S. Fabrication Gallium/Graphene Core-shell Nanoparticles by Pulsed Laser Deposition and their Applications in Surface Enhanced Raman Scattering. Mater. Lett. 2015, 143, 194-196.

(11) Peng, Z.; Somodi, F.; Helveg, S.; Kisielowski, C.; Specht, P.; Bell, A. T. High-Resolution In-Situ and Ex-Situ TEM Studies on Graphene Formation and Growth on Pt Nanoparticles. J. Catal. 2012, 286, 22-29.

(12) Cui, X.; Ren, P.; Deng, D.; Deng, J.; Bao, X. Single Layer Graphene Encapsulating Non-Precious Metals as High-Performance Electrocatalysts for Water Oxidation. Energy Environ. Sci. 2015, 9, 123-129.

(13) Deng, J.; Ren, P.; Deng, D.; Bao, X. Enhanced Electron Penetration Through an Ultrathin Graphene Layer for Highly Efficient Catalysis of the Hydrogen Evolution Reaction. Angew. Chem. Int. Ed. 2015, 54, 2100-2104.

(14) Deng, D.; Novoselov, K. S.; Fu, Q.; Zheng, N.; Tian, Z.; Bao, X. Catalysis with TwoDimensional Materials and their Heterostructures. Nat. Nanotechnol. 2016, 11, 218-230.

(15) Fu, Q.; Bao, X. Surface Chemistry and Catalysis Confined under Two-Dimensional Materials. Chem. Soc. Rev. 2017, 46, 1842-1874. 
(16) Gao, L.; Fu, Q.; Li, J.; Qu, Z.; Bao, X. Enhanced CO Oxidation Reaction over Pt Nanoparticles Covered with Ultrathin Graphitic Layers. Carbon 2016, 101, 324-330.

(17) Wintterlin, J.; Bocquet, M.-L. Graphene on Metal Surfaces. Surface Science 2009, 603, $1841-1852$.

(18) Dahal, A.; Batzill, M. Graphene-Nickel Interfaces: A Review. Nanoscale 2014, 6, $2548-2562$.

(19) Dedkov, Y.; Voloshina, E.; Fonin, M. Scanning Probe Microscopy and Spectroscopy of Graphene on Metals. Phys. Status Solidi B 2015, 252, 451-468.

(20) Murata, Y.; Petrova, V.; Kappes, B. B.; Ebnonnasir, A.; Petrov, I.; Xie, Y.-H.; Ciobanu, C. V.; Kodambaka, S. Moiré Superstructures of Graphene on Faceted Nickel Islands. ACS Nano 2010, 4, 6509-6514.

(21) Rose, M. K.; Borg, A.; Mitsui, T.; Ogletree, D. F.; Salmeron, M. Subsurface Impurities in Pd(111) Studied by Scanning Tunneling Microscopy. J. Chem. Phys. 2001, 115, 10927-10934.

(22) Yudanov, I. V.; Neyman, K. M.; Rösch, N. Density Functional Study of Pd Nanoparticles with Subsurface Impurities of Light Element Atoms. Phys. Chem. Chem. Phys. 2004, 6, 116-123.

(23) Gracia, L.; Calatayud, M.; Andrés, J.; Minot, C.; Salmeron, M. Migration of the Subsurface C Impurity in $\mathrm{Pd}(111)$. Phys. Rev. B 2005, 71, 033407.

(24) Gabasch, H.; Hayek, K.; Klötzer, B.; Knop-Gericke, A.; Schlögl, R. Carbon Incorporation in $\operatorname{Pd}(111)$ by Adsorption and Dehydrogenation of Ethene. J. Phys. Chem. B 2006, 110, 4947-4952. 
(25) Kozlov, S. M.; Yudanov, I. V.; Aleksandrov, H. A.; Rösch, N. Theoretical Study of Carbon Species on Pd(111): Competition between Migration of C Atoms to the Subsurface Interlayer and Formation of Cn Clusters on the Surface. Phys. Chem. Chem. Phys. 2009, 11, 10955.

(26) Nykänen, L.; Andersin, J.; Honkala, K. First-Principles Calculations of the Initial Incorporation of Carbon into Flat and Stepped Pd Surfaces. Phys. Rev. B 2010, 81, 10955.

(27) Wu, P.; Zhang, W.; Li, Z.; Yang, J.; Hou, J. G. Communication: Coalescence of Carbon Atoms on $\mathrm{Cu}(111)$ Surface: Emergence of a Stable Bridging-Metal Structure Motif. $J$. Chem. Phys. 2010, 133, 071101.

(28) Seriani, N.; Mittendorfer, F.; Kresse, G. Carbon in Palladium Catalysts: A Metastable Carbide. J. Chem. Phys. 2010, 132, 024711.

(29) Neyman, K. M.; Schauermann, S. Hydrogen Diffusion into Palladium Nanoparticles: Pivotal Promotion by Carbon. Angew. Chem. Int. Ed. 2010, 49, 4743-4746.

(30) Yokoyama, H.; Numakura, H.; Koiwa, M. The Solubility and Diffusion of Carbon in Palladium. Acta Materialia 1998, 46, 2823-2830.

(31) Sengar, S. K.; Mehta, B. R.; Kumar, R.; Singh, V In-Flight Gas Phase Growth of Metal/Multi Layer Graphene Core Shell Nanoparticles with Controllable Sizes. Sci. Rep. 2013, 3, 2814.

(32) Kwon, S.-Y.; Ciobanu, C. V.; Petrova, V.; Shenoy, V. B.; Bareno, J.; Gambin, V.; Petrov, I.; Kodambaka, S. Growth of Semiconducting Graphene on Palladium. Nano Lett. 2009, 9, 3985-3990. 
(33) Murata, Y.; Starodub, E.; Kappes, B. B.; Ciobanu, C. V.; Bartelt, N. C.; McCarty, K. F.; Kodambaka, S. Orientation-Dependent Work Function of Graphene on Pd(111). Appl. Phys. Lett. 2010, 97, 143114.

(34) Gao, J.-H.; Ishida, N.; Scott, I.; Fujita, D. Controllable Growth of Single-Layer Graphene on a $\operatorname{Pd}(111)$ Substrate. Carbon 2012, 50, 1674-1680.

(35) Murata, Y.; Nie, S.; Ebnonnasir, A.; Starodub, E.; Kappes, B. B.; McCarty, K. F.; Ciobanu, C. V.; Kodambaka, S. Growth Structure and Work Function of Bilayer Graphene on Pd(111). Phys. Rev. B 2012, 85, 1674-1680.

(36) Mok, H. S.; Ebnonnasir, A.; Murata, Y.; Nie, S.; McCarty, K. F.; Ciobanu, C. V.; Kodambaka, S. Kinetics of Monolayer Graphene Growth by Segregation on Pd(111). Appl. Phys. Lett. 2014, 104, 101606.

(37) Gao, J.; Yip, J.; Zhao, J.; Yakobson, B. I.; Ding, F. Graphene Nucleation on Transition Metal Surface: Structure Transformation and Role of the Metal Step Edge. J.Amer. Chem. Soc. 2011, 133, 5009-5015.

(38) Page, A. J.; Wang, Y.; Li, H.-B.; Irle, S.; Morokuma, K. Nucleation of Graphene Precursors on Transition Metal Surfaces: Insights from Theoretical Simulations. J. Phys. Chem. C 2013, 117, 14858-14864.

(39) Giovannetti, G.; Khomyakov, P. A.; Brocks, G.; Karpan, V. M.; van den Brink, J.; Kelly, P. J. Doping Graphene with Metal Contacts. Phys. Rev. Lett. 2008, 101, 026803.

(40) Chapon, C.; Granjeaud, S.; Humbert, A.; Henry, C. R. Structure and Morphology of Nanometer-Sized Pd Clusters Grown at High Temperature on Natural Graphite Single Crystals. Eur. Phys. J. AP 2001, 13, 23-30. 
(41) Gui, G.; Li, J.; Zhong, J. Band Structure Engineering of Graphene by Strain: FirstPrinciples Calculations. Phys. Rev. B 2008, 78, 075435.

(42) Zakharchenko, K. V.; Katsnelson, M. I.; Fasolino, A. Finite Temperature Lattice Properties of Graphene Beyond the Quasiharmonic Approximation. Phys. Rev. Lett. 2009, 102, 046808.

(43) Maeland, A.; Flanagan, T. B. The Hydrogen-Palladium System. Platinum Metals Rev. 1966, 10, 20-24.

(44) Mukhopadhyay, R.; Dasannacharya, B. A.; Nandan, D.; Singh, A. J.; Iyer, R. M. Real Time Deuterium Loading Investigation in Palladium Using Neutron Diffraction. Solid State Commun. 1990, 75, 359-362.

(45) Akiba, H.; Kofu, M.; Kobayashi, H.; Kitagawa, H.; Ikeda, K.; Otomo, T.; Yamamuro, O. Nanometer-Size Effect on Hydrogen Sites in Palladium Lattice. J. Amer. Chem. Soc. 2016, 138, 10238-10243.

(46) Zhang, X.; Xu, Z.; Hui, L.; Xin, J.; Ding, F. How the Orientation of Graphene Is Determined During Chemical Vapor Deposition Growth. J. Phys. Chem. Lett. 2012, 3, $2822-2827$.

(47) Palacios-Lidon, E.; Henry, C. R.; Barth, C. Kelvin Probe Force Microscopy in Surface Chemistry: Reactivity of Pd Nanoparticles on Highly Oriented Pirolytic Graphite. ACS Catal. 2014, 4, 1838-1844.

(48) Barth, C.; Claeys, C.; Henry, C. R. Surface Preparation of Hard Ionic Crystals by Ultrahigh Vacuum Cleavage. Rev. Sci. Instr. 2005, 76, 083907. 
(49) Martín-Recio, A.; Romero-Muñiz, C.; Pou, P.; Pérez, R.; Gómez-Rodríguez, J. M. Purely Substitutional Nitrogen on Graphene/Pt(111) Unveiled by STM and First Principles Calculations. Nanoscale 2016, 8, 17686-17693.

(50) Wang, Z.-J.; Wei, M.; Jin, L.; Ning, Y.; Yu, L.; Fu, Q.; Bao, X. Simultaneous NIntercalation and N-Doping of Epitaxial Graphene on $6 \mathrm{H}-\mathrm{SiC}(0001)$ through Thermal Reactions with Ammonia. Nano Res. 2013, 6, 399-408.

(51) Tison, Y.; Lagoute, J.; Repain, V.; Chacon, C.; Girard, Y.; Rousset, S.; Joucken, F.; Sharma, D.; Henrard, L.; Amara, H. et al. Electronic Interaction between Nitrogen Atoms in Doped Graphene. ACS Nano 2015, 9, 670-678. 


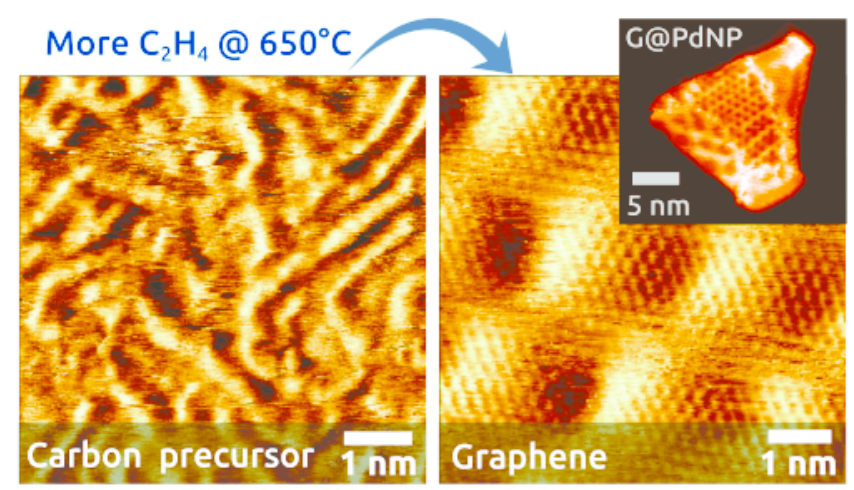

\section{TOC graphic}

